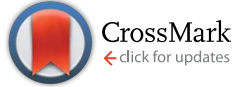

Cite this: RSC Adv., 2015, 5, 61284

Received 31st May 2015

Accepted 9th July 2015

DOI: $10.1039 / c 5 r a 10269 c$

www.rsc.org/advances

\title{
Solar power generation enhancement of dye- sensitized solar cells using hydrophobic and antireflective polymers with nanoholes
}

\author{
Joo Ho Lim, Jung Woo Leem and Jae Su Yu*
}

\begin{abstract}
We improve the power conversion efficiency (PCE) of dye-sensitized solar cells (DSSCs) using polydimethylsiloxane (PDMS) patterned with negatively tapered nanoholes (NHs) as a protective antireflection layer of the external glass surface. The NHs PDMS layers are prepared by a soft lithography via silicon molds with conical nanopillars. The NHs PDMS with a $\mathrm{NH}$ depth of $\sim 320 \mathrm{~nm}$ decreases the surface reflection of fluorine doped tin oxide (FTO)-coated glass over a wide wavelength range of $350-800 \mathrm{~nm}$ at incident angles $\left(\theta_{\text {in }}\right)$ of $0-70^{\circ}$, exhibiting a lower solar weighted reflectance $\left(R_{\text {SW }}\right)$ value of $\sim 7.1 \%$ at $\theta_{\text {in }}=0^{\circ}$ and a lower average $R_{\mathrm{SW}}$ value of $\sim 8.5 \%$ at $\theta_{\text {in }}=20-70^{\circ}$ than those (i.e., $R_{\mathrm{SW}} \approx$ $10.1 \%$ at $\theta_{\text {in }}=0^{\circ}$ and average $R_{\mathrm{SW}} \approx 15.6 \%$ at $\theta_{\text {in }}=20-70^{\circ}$ ) of the FTO glass. In DSSC device applications, it increases the short-circuit current density $\left(J_{\mathrm{SC}}\right)$ from 15.69 to $16.52 \mathrm{~mA} \mathrm{~cm}{ }^{-2}$, thus resulting in an enhanced PCE value of $7.56 \%$ compared to the reference DSSC (i.e., PCE $=7.15 \%$ ). For different NH depths, the optical reflectance characteristics of the NHs PDMS/FTO glass are theoretically investigated using a rigorous coupled-wave analysis method, showing similar trends between the calculated and measured results. For solar spectrum angle-dependent photocurrents, it also shows a remarkable device performance at $\theta_{\text {in }}=20-70^{\circ}$. Besides, the NHs PDMS exhibits a hydrophobic surface with a water contact angle of $\sim 115^{\circ}$.
\end{abstract}

\section{Introduction}

Over the past decades, research into alternative energy sources has been a global issue as a result of enhanced environmental pollution and fossil-fuel consumption. Solar energy, among several other alternative energy sources, has been attracting interest in various application fields because of its abundance, cost-effective production, and environmental friendliness. In particular, comparing the dye-sensitized solar cell (DSSC) with other solar cells, it has some advantages such as low manufacturing cost, lightweight, flexibility, transparency, and so on. ${ }^{1-3}$ However, the power conversion efficiencies (PCEs) of DSSCs fall below 13\%, mainly due to limitations including the light absorption of the dyes and the interface charge separation between dyes and titanium dioxide $\left(\mathrm{TiO}_{2}\right)$ electrodes. ${ }^{2,3}$ Hence, possibilities exist for further enhancements in PCEs of DSSCs. In order to boost the light absorption in the dyed $\mathrm{TiO}_{2}$ layer of the devices, among various approaches for improving the PCE, one simple method is the employment of efficient antireflection coatings (ARCs) on the front surface of the substrates. As an alternative of conventional ARCs consisting of single layer or multilayers, recently, many studies have been reported on the

Department of Electronics and Radio Engineering, Institute for Wearable Convergence Electronics, Kyung Hee University, 1732 Deogyeong-daero, Giheung-gu, Yongin-si 446-701, South Korea. E-mail: jsyu@khu.ac.kr nanostructured surfaces (e.g., nanogratings, nanorods, and nanoporous films) on external substrates or covers of solar cells as ARCs. ${ }^{4-7}$ However, to directly fabricate the nanostructures on substrates, nanopatterning (e.g., electron-beam, nanoimprint, or laser interference lithography) and etching processes including chemical and heat treatments are required, which increase the fabrication cost and complexity. Therefore, it is necessary to develop efficient ARCs of glasses in the effective ways including cost-effective, simple, and high-throughput processes. In this respect, soft lithography, which has been widely utilized to form the various nanostructures onto the surface of polymers, can be one of them. ${ }^{8-11}$ The fabricated molds and stamps can be also repeatedly used for pattern transfer, and thus would facilitate the mass production (i.e., roll-to-roll or roll-to-plate) in industry applications. In the soft lithography, meanwhile, the dimethylsiloxane (PDMS) is generally used as a protective encapsulation layer against mechanical damage to the underlying devices due to its low free surface energy, flexibility, transparency, and hardness. ${ }^{\mathbf{1 0 - 1 4}}$ Besides, it can increase the optical transmission of transparent substrates including glasses, polymers, etc. because of its lower refractive index of $\sim 1.4-1.43 .{ }^{14,15}$ Furthermore, the PDMS layer can be strongly attached on the surface of planar transparent substrates with detachability from it. On the other hand, the hydrophobicity is useful in outdoor applications because it can function the self-cleaning for the pollutants and dusts on the 
surface of devices. ${ }^{\mathbf{1 6}, \mathbf{1 7}}$ Thus, it is very meaningful to investigate the optical and wetting behaviors of nanostructured PDMS layers as well as their feasibility on the device performance of DSSCs. In this work, we demonstrated the PCE enhancement of DSSCs via protective AR PDMS layers with nanoholes (NHs) patterns. The NHs on the PDMS layers were negatively transferred from the silicon ( $\mathrm{Si}$ ) molds with tapered nanopillars by the soft lithography. Their optical reflectance characteristics, together with a theoretical prediction using a rigorous coupledwave analysis (RCWA) method, were also studied at various incident angles of light, including the surface wettability.

\section{Experimental and numerical modelling details}

\section{Fabrication of NHs PDMS and DSSCs}

Fig. 1 shows the schematic diagram for the fabrication procedure of the NHs-patterned PDMS (i.e., NHs PDMS) from the Si mold with tapered nanopillars via the soft lithography. The conical nanopillars on Si molds were prepared by using the thermally dewetted platinum (Pt) nanoparticles as an etch mask pattern and subsequent dry etching process, as reported in our previous work. ${ }^{18}$ As shown in Fig. 1, to fabricate the NHs on the surface of PDMS layers without any distortion and deformation, we performed two-step coating processes. Firstly, a mixture solution consisting of the trimethylsiloxy terminated vinylmethylsiloxane-dimethylsiloxane (VDT-731; Gelest, Inc.) and methylhydrosiloxane-dimethylsiloxane (HMS-301; Gelest, Inc.) copolymers was spin-coated on the Si molds, and then they were cured at a temperature of $75^{\circ} \mathrm{C}$ for $30 \mathrm{~min}$. Afterwards, the Sylgard 184 PDMS (base : agent $=10: 1$ ) solution was poured on the samples with curing at $75{ }^{\circ} \mathrm{C}$ for $2 \mathrm{~h}$. Finally, the PDMS layers were carefully separated from the Si molds, creating the NHs PDMS. To study the effect of depth of NHs on the optical reflectance property, the NHs PDMS samples with NH depths of 150 and $320 \mathrm{~nm}$ were prepared, named NHs-A at $150 \mathrm{~nm}$ and NHs-B at $320 \mathrm{~nm}$, respectively. On the other hand, DSSCs were made by a conventional fabrication process. A $\mathrm{TiO}_{2}$ paste was deposited on fluorine doped tin oxide (FTO)-coated glasses using a doctor-blade method. Then, it was annealed at $500{ }^{\circ} \mathrm{C}$ for $2 \mathrm{~h}$ in a furnace. The deposition and annealing processes were repeated to fix the thickness of the deposited $\mathrm{TiO}_{2}$ paste by $\sim 15 \mu \mathrm{m}$. For the ruthenium(II) dye (Solaronix, N719), the $\mathrm{TiO}_{2}$ coated FTO glass substrates were immersed in a $3 \times 10^{-4} \mathrm{M}$ solution with mixing dye for $24 \mathrm{~h}$ in dark state. By using the same doctor-blade process, a Pt paste (Dyesol, counter PT-1) as a counter electrode was coated onto the FTO glass substrates. Lastly, an electrolyte (Dyesol, electrolyte HPD) was injected, and thus the DSSCs with an active area of $0.25 \mathrm{~cm}^{2}$ were fabricated.

\section{Characterization instruments}

A scanning electron microscope (SEM; LEO SUPRA 55, Carl Zeiss) was utilized for observing the surface morphologies and pattern profiles of the fabricated samples. The surface wetting behaviors were evaluated by using a contact angle measurement system (Phoenix-300, SEO). A UV-vis-NIR spectrophotometer

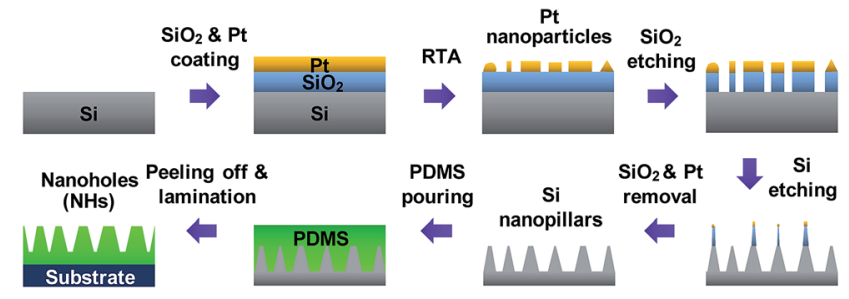

Fig. 1 Schematic diagram for the fabrication procedure of the nanoholes-patterned PDMS (i.e., NHs PDMS) from the Si mold with tapered nanopillars via the soft lithography.

(Cary 5000, Varian) was used to characterize optical reflectance properties. The current density-voltage $(J-V)$ characteristics of DSSCs were measured by using a photocurrent system consisting of a solar simulator (ABET, SUN 3000) with $1000 \mathrm{~W}$ Xe short arc lamp and a source meter (Keithley 2400). Incident photon to current conversion efficiency (IPCE) data were obtained by illuminating monochromatic light on the DSSCs using a $300 \mathrm{~W}$ xenon arc lamp as the light source coupled to a monochromator (TLS-300 $\times$ xenon light source, Newport) with optical power meter (2935-c, Newport).

\section{Numerical modelling and simulations}

The theoretical analysis on the optical reflectance and the electric field intensity distribution of the NHs PDMS were also studied by the RCWA method using a commercial software (DiffractMOD, Rsoft Design Group). To design the numerical model, the shape of the negatively tapered nanohole structure (i.e., empty space, air) on the surface of the PDMS was represented by a periodic geometry in the Cartesian coordinate system using a scalar-valued function of three variables, $f(x, y$, $z$ ), for simplicity. We assumed that the incident light enters from air to the PDMS layer. The $E_{y}$, i.e., amplitude of $y$-polarized electric field, was calculated for the incident plane wave with a Gaussian beam profile that is normalized at $\lambda=530 \mathrm{~nm}$. The period of NHs was kept at $280 \mathrm{~nm}$. The thicknesses of constituent layers were set to be $150 \mu \mathrm{m}$ for the PDMS, $500 \mu \mathrm{m}$ for the glass, and $650 \mathrm{~nm}$ for the FTO film, respectively. The calculated values at each wavelength were averaged to remove rapid fluctuations caused by the interference of light reflected at the top and bottom surfaces of the PDMS/glass/FTO. The refractive index and extinction coefficient of glass and FTO were acquired from index Web site. ${ }^{19}$ For the PDMS, the refractive index was assumed to be 1.43 .

\section{Results and discussion}

Fig. 2 shows (a) the $40^{\circ}$-tilted oblique-view SEM images of the $\mathrm{Si}$ mold with nanopillars and the fabricated PDMS with nanoholes (NHs) and (b) the photographs of a water droplet on the surface of corresponding PDMS samples. Using the soft lithography technique, the negatively tapered nanohole arrays were relatively well transferred onto the surface of the PDMS films from conical nanopillar-structured Si molds (i.e., average height $\approx 170 \mathrm{~nm}$ for the nanopillars-A and $\approx 380 \mathrm{~nm}$ for the 

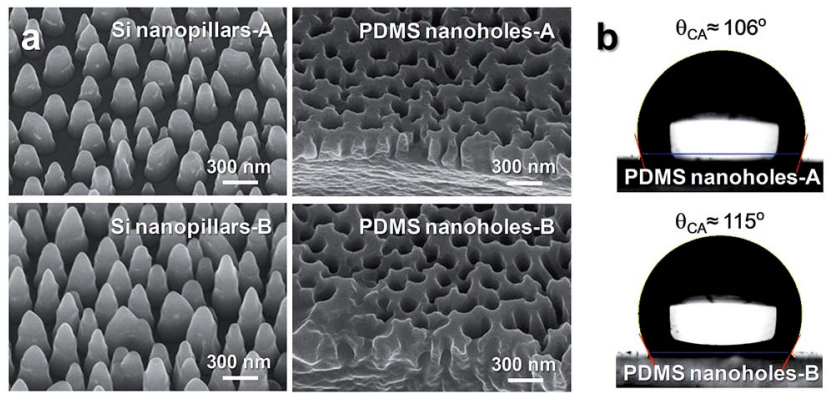

Fig. 2 (a) $40^{\circ}$-tilted oblique-view SEM images of the Si mold with nanopillars and the fabricated PDMS with $\mathrm{NHs}$ and (b) photographs of a water droplet on the surface of corresponding PDMS samples.

nanopillars-B) without any large deformation and distortion, as shown in the SEM images of Fig. 2(a). For the formed NHs on the PDMS films, the average $\mathrm{NH}$ depths were estimated to be $\sim 150 \mathrm{~nm}$ (i.e., NHs-A PDMS) and $2320 \mathrm{~nm}$ (i.e., NHs-B PDMS), respectively. The PDMS with a flat surface showed a hydrophobic property with a water contact angle $\left(\theta_{\mathrm{CA}}\right)$ of $\sim 99^{\circ} .^{20}$ The introduction of the roughness on the surface enhances the hydrophobicity, as can be explained by the Cassie-Baxter theory. ${ }^{21}$ Thus, the larger $\theta_{\mathrm{CA}}$ values were obtained for the NHs
PDMS, exhibiting the $\theta_{\mathrm{CA}} \approx 106^{\circ}$ for the NHs-A and $\theta_{\mathrm{CA}} \approx 115^{\circ}$ for the NHs-B, respectively. This hydrophobic surface might self-clean the dusts and contaminants on the devices in outdoor environments.

Fig. 3 shows (a) the measured reflectance spectra of the FTO glass, the flat PDMS/FTO glass, the NHs-A PDMS/FTO glass, and the NHs-B PDMS/FTO glass, (b) the contour plot of the variation of the calculated reflectance spectra of NHs PDMS/FTO glass as functions of wavelength and depth of NHs, and (c) the electric field $\left(E_{y}\right)$ intensity distributions for the corresponding structures at $\lambda=530 \mathrm{~nm}$. As shown in Fig. 3(a), by laminating the flat PDMS on the glass surface of the FTO glass, the surface reflectance of the FTO glass was reduced because of the step gradient refractive index (GRIN) profile in constituent materials of air ( $n=1) /$ PDMS (1.43)/glass (1.52)/FTO (1.98). On the other hand, the NHs PDMS further decreased the reflectance over a wide wavelength range of $350-800 \mathrm{~nm}$. This is attributed to the linear and continuous GRIN profile via negatively tapered NHs (i.e., moth-eye effect). ${ }^{22}$ For the NHs-B PDMS with a larger NH depth of $\sim 320 \mathrm{~nm}$, its solar weighted reflectance $\left(R_{\mathrm{SW}}\right)$ value of $\sim 7.1 \%$, which is the ratio of the usable photons reflected to the total usable photons, ${ }^{23}$ at wavelengths of $350-800 \mathrm{~nm}$ is lower than those of the other samples (i.e., $R_{\mathrm{SW}} \approx 10.1 \%$, $9 \%$, and $7.9 \%$ for
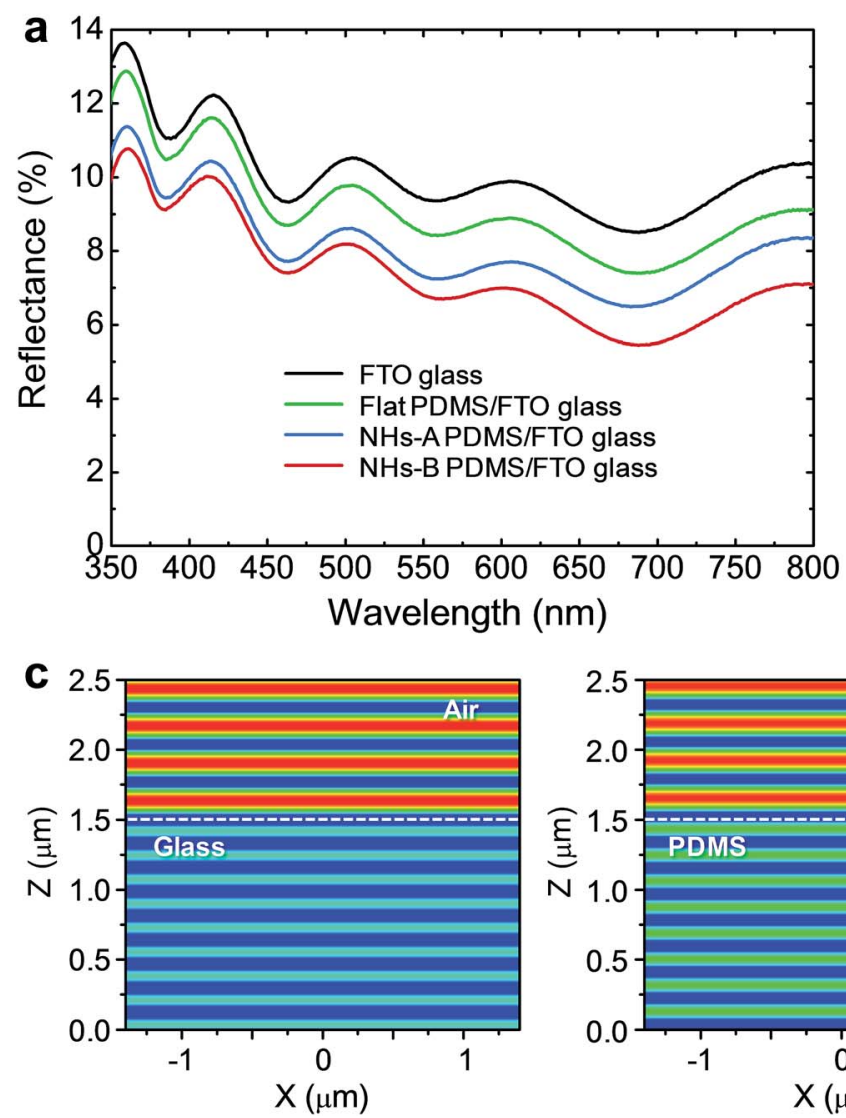
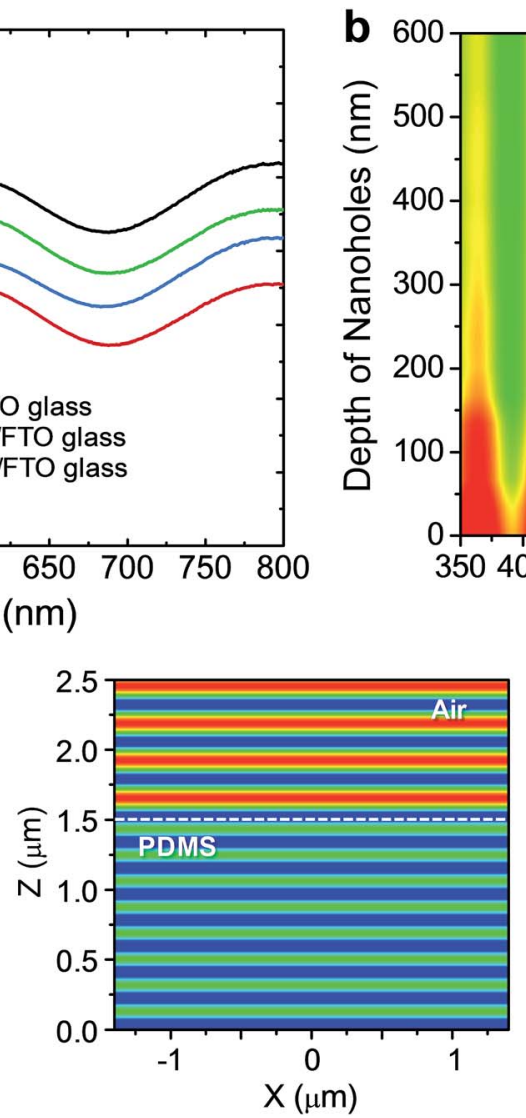

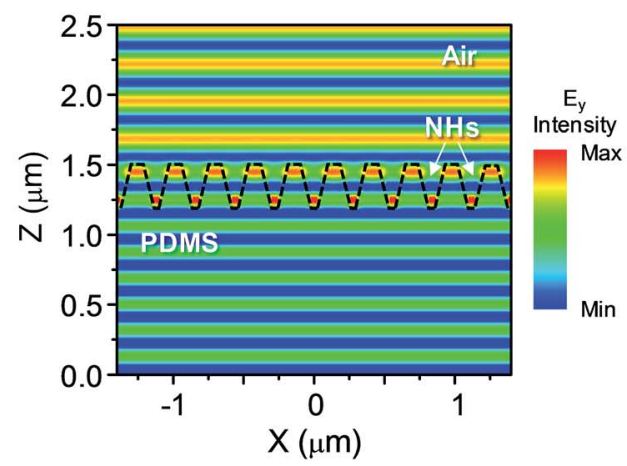

Fig. 3 (a) Measured reflectance spectra of the FTO glass, flat PDMS/FTO glass, NHs-A PDMS/FTO glass, and NHs-B PDMS/FTO glass, (b) contour plot of the variation of the calculated reflectance spectra of NHs PDMS/FTO glass as functions of wavelength and depth of NHs, and (c) $E_{y}$ intensity distributions for the corresponding structures at $\lambda=530 \mathrm{~nm}$. The $R_{\mathrm{SW}}$ values of NHs PDMS/FTO glass as a function of depth of NHs are also shown in the inset of (b). 

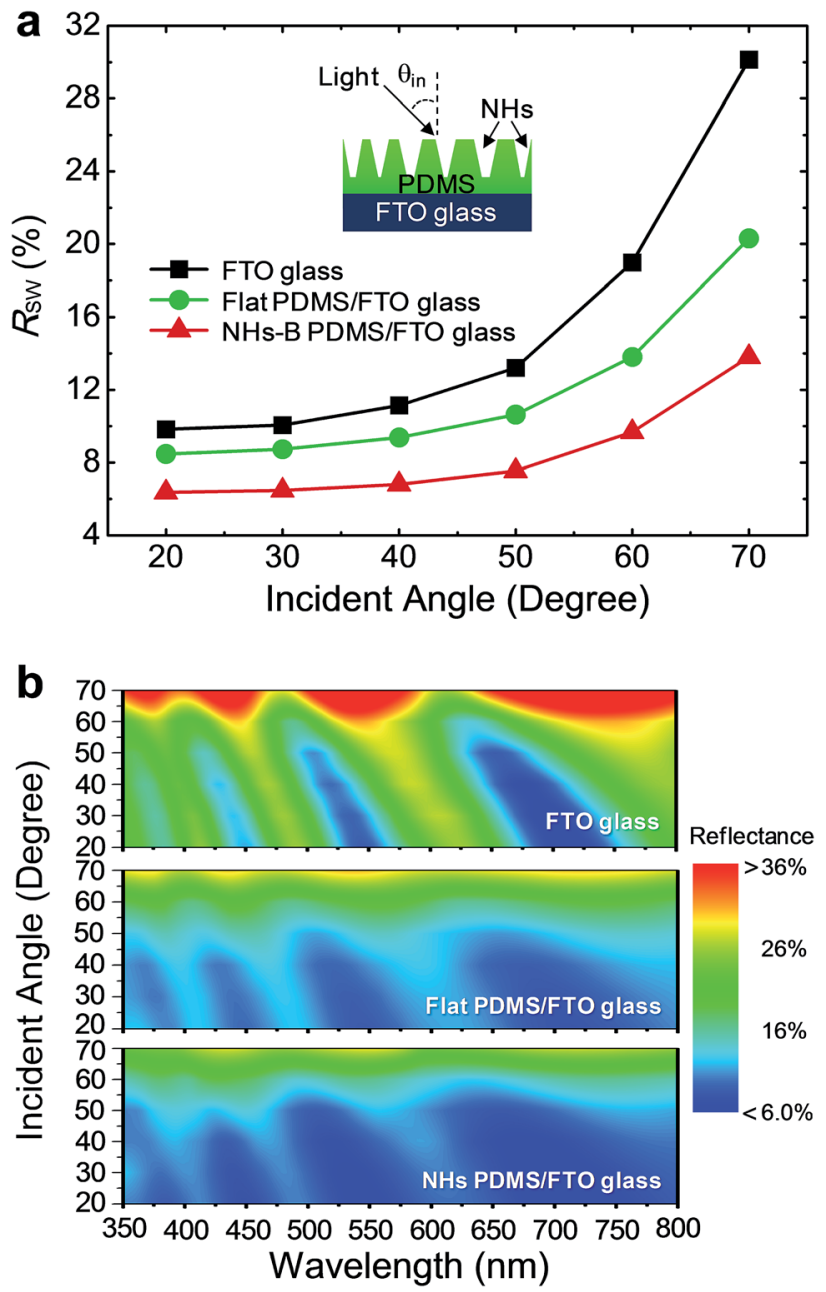

Fig. 4 (a) $R_{\mathrm{SW}}$ values for the measured reflectance spectra of the FTO glass, the flat PDMS/FTO glass, and the NHs-B PDMS/FTO glass as a function of incident angle $\left(\theta_{\text {in }}\right)$ in the wavelength range of 350-800 $\mathrm{nm}$ and (b) contour plots of variations of the calculated angledependent reflectance spectra for the corresponding structures.

the FTO glass, the flat PDMS/FTO glass, and the NHs-A PDMS/FTO glass, respectively). In RCWA simulations of Fig. 3(b), the calculated reflectance results of NHs PDMS at different depths of NHs were roughly similar to the experimentally measured spectra in Fig. 3(a). The $R_{\mathrm{SW}}$ values of NHs PDMS/FTO glass as a function of depth of NHs are also shown in the inset of Fig. 3(b). The $R_{\mathrm{SW}}$ values are abruptly decreased from $9.6 \%$ at $0 \mathrm{~nm}$ (i.e., flat PDMS/FTO glass) to $7.6 \%$ at $200 \mathrm{~nm}$ and they are minimally saturated at $\mathrm{NH}$ depths of $>300 \mathrm{~nm}$, exhibiting $R_{\text {SW }}$ values of $\sim 7.4-7.5 \%$. These results show a similar tendency with the optical properties of transparent materials (e.g., glasses, polymers, etc.) with one-side antireflective nanostructured surface reported in other previous works. ${ }^{24-26}$ Therefore, it can be considered that the NHs PDMS with the NH depth of $\sim 320 \mathrm{~nm}$ is an optimized sample that has the lowest $R_{\mathrm{SW}}$ value of $\sim 7.1 \%$ in the NHs PDMS/FTO glass structure. Additionally, the antireflection characteristics of the NHs PDMS can be observed in the calculated $E_{y}$ intensity distributions. In Fig. 3(c), for both the glass and flat PDMS, the
$E_{y}$ intensities at $\lambda=530 \mathrm{~nm}$ are high in the air region due to their strong surface reflection. For the NHs PDMS, on the other hand, there exist relatively weaker $E_{y}$ intensities in the air region due to the suppressed reflectivity caused by the GRIN profile of the NHs on the surface of PDMS.

It is also important to study incident light angle-dependent reflectance characteristics due to the change of the sun position in a day and seasons. Fig. 4 shows (a) the $R_{\mathrm{SW}}$ values for the measured reflectance spectra of the FTO glass, the flat PDMS/FTO glass, and the NHs-B PDMS/FTO glass as a function of incident angle $\left(\theta_{\text {in }}\right)$ in the wavelength range of $350-800 \mathrm{~nm}$ and (b) the contour plots of variations of the calculated angledependent reflectance spectra for the corresponding structures. As shown in Fig. 4(a), for all the samples, the $R_{\mathrm{SW}}$ values gradually increased with increasing the $\theta_{\text {in }}$ value from 20 to $70^{\circ}$. However, as expected, the NHs-B PDMS with a superior AR ability further decreased the $R_{\mathrm{SW}}$ values of FTO glass compared to the flat PDMS at each $\theta_{\text {in }}$ value, exhibiting a lower average $R_{\mathrm{SW}}$ value of $\sim 8.5 \%$ at $\theta_{\text {in }}=20-70^{\circ}$ (i.e., average $R_{\mathrm{SW}}$ values of $\sim 15.6 \%$ and $\sim 11.9 \%$ for the FTO glass and the flat PDMS/FTO
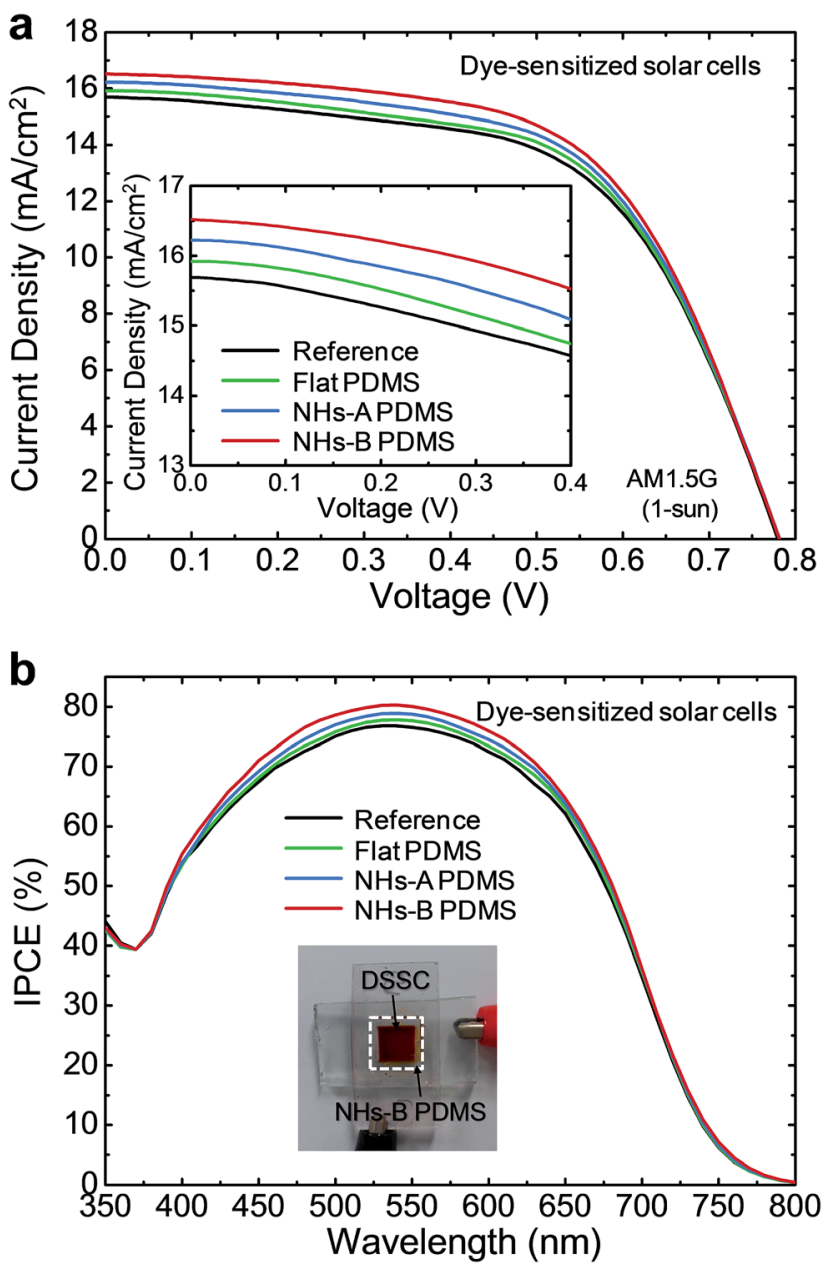

Fig. 5 (a) $J-V$ curves and (b) IPCE spectra of the reference DSSC without PDMS and the DSSCs with the flat PDMS, the NHs-A PDMS, and the NHs-B PDMS. Photograph of the DSSC incorporated with NHs-B PDMS protective AR layer is also shown in the inset of (b). 
Table 1 Device characteristics of the corresponding DSSCs

\begin{tabular}{lllll}
\hline DSSC & $V_{\mathrm{OC}}(\mathrm{V})$ & $J_{\mathrm{SC}}\left(\mathrm{mA} \mathrm{cm}^{-2}\right)$ & FF $(\%)$ & PCE $(\%)$ \\
\hline Reference & 0.780 & 15.69 & 58.44 & 7.15 \\
Flat PDMS & 0.780 & 15.92 & 58.76 & 7.30 \\
NHs-A PDMS & 0.781 & 16.22 & 58.68 & 7.44 \\
NHs-B PDMS & 0.781 & 16.52 & 58.62 & 7.56
\end{tabular}

glass, respectively). Similarly, in the RCWA calculations of Fig. 4(b), the NHs PDMS/FTO glass shows the less-angle dependent reflectance characteristics compared to the other structures in wide ranges of incident wavelengths and angles.

In order to demonstrate the AR effect of the NHs PDMS in DSSCs, the device characteristics of DSSCs employed with NHs PDMS as a protective AR layer were investigated. Fig. 5 shows the (a) $J-V$ curves and (b) IPCE spectra of the reference DSSC without PDMS and the DSSCs with the flat PDMS, the NHs-A PDMS, and the NHs-B PDMS. Photograph of the DSSC incorporated with NHs-B PDMS protective AR layer is also shown in the inset of Fig. 5(b). It can be observed that the PDMS is well laminated on the external glass surface of the DSSC due to van der Waals force between the PDMS and glass. The device characteristics of the corresponding DSSCs are summarized in Table 1. As shown in Fig. 5(a), by laminating the PDMS on the glass surface of DSSCs, the increased short-circuit current density $\left(J_{\text {sC }}\right)$ values were observed while there was no large variation in both the open-circuit voltage $\left(V_{\mathrm{OC}}\right)$ and fill factor (FF). The DSSC with the NHs-B PDMS especially exhibited a higher $J_{\mathrm{SC}}$ value of $16.52 \mathrm{~mA} \mathrm{~cm}^{-2}$ than those of the other DSSCs (i.e., $J_{\mathrm{SC}}=15.69,15.92$, and $16.22 \mathrm{~mA} \mathrm{~cm}^{-2}$ for the reference DSSC and the DSSCs with the flat PDMS and the NHs-A PDMS, respectively). This is ascribed to the reduced surface reflection by the linear GRIN profile via NHs on the PDMS in Fig. 3(a), as mentioned above. The increased photocurrent can be also confirmed in the IPCE data of Fig. 5(b). The DSSC with the

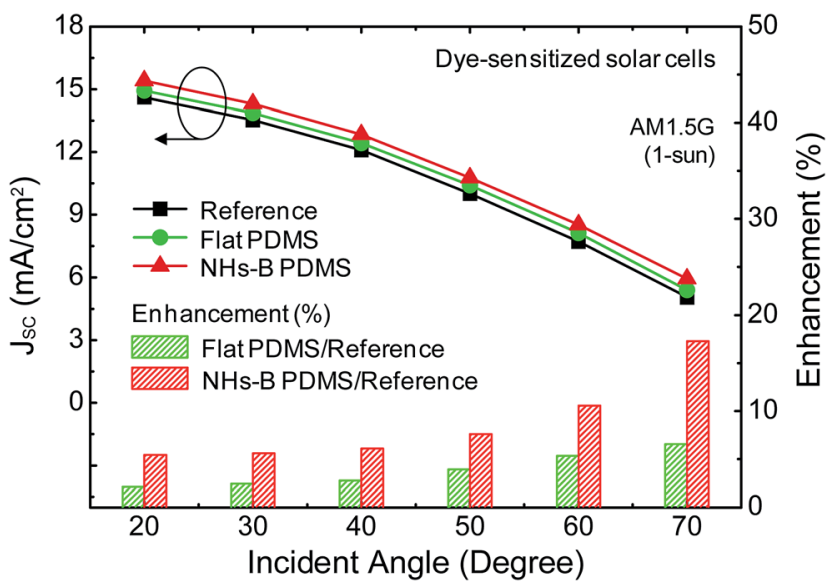

Fig. $6 J_{S C}$ of values the reference DSSC and the DSSCs with the flat PDMS, and the NHs-B PDMS and estimated enhancement percentage in $J_{S C}$ for the DSSCs with the flat PDMS DSSC and the NHs PDMS relative to the reference DSSC at $\theta_{\text {in }}=20-70^{\circ}$.
NHs-B PDMS showed a higher IPCE spectrum compared to the other DSSCs in the wavelength range of $400-800 \mathrm{~nm}$. As a result, the PCE value of the DSSC with the NHs-B PDMS was improved by $7.56 \%$ (i.e., PCE $=7.15 \%$ for the reference DSSC), indicating the PCE enhancement percentage of $\sim 5.8 \%$.

To study the angle-dependence of solar power generation in DSSCs with the AR NHs PDMS, their $J_{\mathrm{SC}}$ values were measured and the enhancement percentage in $J_{\mathrm{SC}}$ was estimated for the DSSC with the flat PDMS and the NHs-B PDMS relative to the reference DSSC at $\theta_{\text {in }}=20-70^{\circ}$, as shown in Fig. 6 . For all the DSSCs, the $J_{\mathrm{SC}}$ values were generally reduced with increasing the $\theta_{\text {in }}$ value. This is attributed to the reduction of the projection area where the incident light enters into the DSSC due to the tilted DSSC from the normally incident light source of the solar simulator as well as the increased surface reflection losses. However, the DSSC with the NHs-B PDMS showed the higher $J_{\mathrm{SC}}$ value at each $\theta_{\text {in }}$ value, maintaining a larger average enhancement percentage value of $\sim 8.8 \%$ compared to the reference DSSC at $\theta_{\text {in }}=20-70^{\circ}$ (i.e., $\sim 3.9 \%$ for the flat PDMS DSSC/ reference DSSC). Thus, the use of the NHs PDMS AR layer with $\mathrm{NH}$ depths of $>300 \mathrm{~nm}$ can lead to the enhancement of the solar energy harvesting in DSSCs for an entire day and the seasons.

\section{Conclusions}

The inversely tapered NHs were fabricated on the surface of the PDMS by the soft lithography via the Si mold with conical nanopillars and their optical reflectance properties were experimentally and theoretically investigated. For the $320 \mathrm{~nm}$-depth NHs-B PDMS/FTO glass with the hydrophobic surface (i.e., $\theta_{\mathrm{CA}}$ $\approx 115^{\circ}$ ), it showed the superior AR characteristics at wavelengths of 350-800 nm and $\theta_{\text {in }}=0-70^{\circ}$, exhibiting the lower $R_{\mathrm{SW}}$ $\approx 7.1 \%$ at $\theta_{\text {in }}=0^{\circ}$ and average $R_{\mathrm{SW}} \approx 8.5 \%$ at $\theta_{\text {in }}=20-70^{\circ}$. By incorporating the NHs-B PDMS into the outer glass of DSSCs as a protective AR layer, the $J_{\mathrm{SC}}$ was not only enhanced to $16.52 \mathrm{~mA}$ $\mathrm{cm}^{-2}$, but also the IPCE spectrum was increased, thus leading to the improved PCE value of $7.56 \%$ (i.e., $\mathrm{PCE}=7.15 \%$ for the reference DSSC). For incident light angle-dependent photocurrents, it also showed a superior solar power generation ability at $\theta_{\text {in }}=20-70^{\circ}$. These results can provide a deep insight into the nanostructured PDMS for omnidirectional broadband ARCs in various photovoltaic systems that use transparent substrates and covers including glasses and plastics.

\section{Acknowledgements}

This work was supported by the National Research Foundation of Korea (NRF) grant funded by the Korea government (MSIP) (No. 2014-069441).

\section{References}

1 B. O'Regan and M. Grätzel, Nature, 1991, 353, 737.

2 S. Mathew, A. Tella, P. Gao, R. Humphry-Baker, B. F. E. Curchod, N. Ashari-Astani, I. Tavernelli, 
U. Rothlisberger, M. K. Nazeeruddin and M. Grätzel, Nat. Chem., 2014, 6, 242.

3 E. Palomares, J. N. Clifford, S. A. Haque, T. Lutz and J. R. Durrant, J. Am. Chem. Soc., 2003, 125, 475.

4 M. L. Brongersma, Y. Cui and S. Fan, Nat. Mater., 2014, 13, 451.

5 Y. C. Wang, B. Y. Lin, P. T. Liu and H. P. D. Shieh, Opt. Express, 2014, 22, A13.

6 Y. Zhang, C. Zhao, P. Wang, L. Ye, J. Luo and B. Jiang, Chem. Commun., 2014, 50, 13813.

7 Y. M. Song, E. S. Choi, G. C. Park, C. Y. Park, S. J. Jang and Y. T. Lee, Appl. Phys. Lett., 2010, 97, 093110.

8 J. Zhang, S. She, X. X. Dong and L. S. Chen, Opt. Express, 2014, 22, 1842.

9 J. W. Leem, M. Choi and J. S. Yu, ACS Appl. Mater. Interfaces, 2015, 7, 2349.

10 Y. M. Song, Y. Xie, V. Malyarchuk, J. Xiao, I. Jung, K. J. Choi, Z. Liu, H. Park, C. Lu, R. H. Kim, R. Li, K. B. Crozier, Y. Huang and J. A. Rogers, Nature, 2013, 497, 95.

11 M. Choi, J. W. Leem and J. S. Yu, RSC Adv., 2015, 5, 25616.

12 T. Bhuvana, B. Kim, X. Yang, H. Shin and E. Kim, Angew. Chem., Int. Ed., 2013, 52, 1180.

13 K. Bao, X. N. Kang, B. Zhang, T. Dai, C. Xiong, H. Ji, G. Y. Zhang and Y. Chen, IEEE Photonics Technol. Lett., 2007, 19, 1840.
14 F. Galeotti, F. Trespidi, G. Timò and M. Pasini, ACS Appl. Mater. Interfaces, 2014, 6, 5827.

15 S. M. Azmayesh-Fard, L. Lam, A. Melnyk and R. G. DeCorby, Opt. Express, 2013, 21, 11889.

16 J. Zhu, C. M. Hsu, Z. Yu, S. Fan and Y. Cui, Nano Lett., 2010, 10, 1979.

17 Y. B. Park, H. Im, M. Im and Y. K. Choi, J. Mater. Chem., 2011, 21, 633.

18 J. W. Leem, K. S. Chung and J. S. Yu, Curr. Appl. Phys, 2012, 12, 291.

19 SOPRA, N\&K Database (2015), retrieved 2015, <http:// refractiveindex.info $>$.

20 J. H. Lim, Y. H. Ko, J. W. Leem and J. S. Yu, Opt. Express, 2015, 23, A169.

21 A. B. D. Cassie and S. Baxter, Trans. Faraday Soc., 1944, 40, 546.

22 D. G. Stavenga, S. Foletti, G. Palasantzas and K. Arikawa, Proc. R. Soc. A, 2006, 273, 661.

23 J. W. Leem, Y. M. Song and J. S. Yu, Nanoscale, 2013, 5, 10455.

24 Y. M. Song, H. J. Choi, J. S. Yu and Y. T. Lee, Opt. Express, 2010, 18, 13063.

25 K. Choi, S. H. Park, Y. M. Song, Y. T. Lee, C. K. Hwangbo, H. Yang and H. S. Lee, Adv. Mater., 2010, 22, 3713.

26 J. W. Leem, Y. Yeh and J. S. Yu, Opt. Express, 2012, 20, 4056. 